\title{
ARTICLE
}

\section{Microwave spectroscopic observation of distinct electron solid phases in wide quantum wells}

\author{
A.T. Hatke1, Yang Liu², B.A. Magill', B.H. Moon¹, L.W. Engel' ${ }^{1}$ M. Shayegan², L.N. Pfeiffer², K.W. West ${ }^{2}$ \\ \& K.W. Baldwin ${ }^{2}$
}

In high magnetic fields, two-dimensional electron systems can form a number of phases in which interelectron repulsion plays the central role, since the kinetic energy is frozen out by Landau quantization. These phases include the well-known liquids of the fractional quantum Hall effect, as well as solid phases with broken spatial symmetry and crystalline order. Solids can occur at the low Landau-filling termination of the fractional quantum Hall effect series but also within integer quantum Hall effects. Here we present microwave spectroscopy studies of wide quantum wells that clearly reveal two distinct solid phases, hidden within what in d.c. transport would be the zero diagonal conductivity of an integer quantum-Hall-effect state. Explanation of these solids is not possible with the simple picture of a Wigner solid of ordinary (quasi) electrons or holes.

\footnotetext{
${ }^{1}$ National High Magnetic Field Laboratory, Tallahassee, Florida 32310, USA. ${ }^{2}$ Department of Electrical Engineering, Princeton University, Princeton, New Jersey 08544, USA. Correspondence and requests for materials should be addressed to A.T.H. (email: hatke@magnet.fsu.edu).
} 
S olid phases ${ }^{1-8}$ of carriers are insulators, owing to pinning by disorder, and are not easily distinguishable from other types of insulators by standard d.c. transport measurements, but rather by a characteristic resonance, at frequency $f_{\mathrm{pk}}$ in their microwave conductivity spectra ${ }^{5,9-11}$. These resonances are understood as pinning modes ${ }^{5,11}$ in which pieces of the solid oscillate within the disorder potential, as diagrammed in Fig. 1a. Pinning modes have been found both near the low Landau filling $(v)$ termination of the fractional quantum Hall effect (FQHE) series $^{5,10}$ and within the $v$ ranges of integer quantum Hall effect (IQHE) plateaus ${ }^{9}$. In the weak pinning picture ${ }^{12-14}, f_{\mathrm{pk}}$ increases when the shear modulus, $C_{\mathrm{t}}$, decreases, for example, by decreasing the density, $n$ (ref. 15). The inverse relation of $f_{\mathrm{pk}}$ and $C_{\mathrm{t}}$ is because carriers associate more closely with minima in the disorder potential ${ }^{12-14}$. Consistent with this picture, in wells narrower than those studied here ${ }^{9}$, a solid within the IQHE range shows a monotonic decrease in $f_{\mathrm{pk}}$ as $v$ moves away from the quantizing filling, which increases the charge density of the solid.

In the following, we report pinning modes whose $f_{\mathrm{pk}}$ exhibits an upward step as $v$ is decreased from 1, in contrast to the monotonic decrease in $f_{\mathrm{pk}}$ that was seen in narrower wells. The phenomenon is seen in wide quantum wells (WQWs) ${ }^{16-20}$, in which the effective electron-electron interaction is softened at short range due to the large growth-direction extent of the wavefunction. A wider well, or a larger $n$, causes the step to move closer to $v=1$. We provide a natural interpretation, based on the sensitivity of $f_{\mathrm{pk}}$ to the properties of the solid, that the step signals a transition between different solids.

\section{Results}

Sample details. Our samples are from wafers containing GaAs quantum wells of widths $w=54$ and $65 \mathrm{~nm}$. For both widths, the quantum wells were flanked on either side by undoped $\mathrm{Al}_{0.24} \mathrm{Ga}_{0.76}$ As. Henceforth, in this paper all $n$ will be in units of $10^{11} \mathrm{~cm}^{-2}$ for brevity. The $54 \mathrm{~nm}$ well has depth of the two dimensional electron system (2DES) from the top $d=430 \mathrm{~nm}$, as-cooled $n=2.42$ and mobility $\mu=5.7 \times 10^{6} \mathrm{~cm}^{2} \mathrm{~V}^{-1} \mathrm{~s}^{-1}$ at $300 \mathrm{mK}$. The $65 \mathrm{~nm}$ well has $d=510 \mathrm{~nm}$, as-cooled $n=1.52$, and $\mu=5.2 \times 10^{6} \mathrm{~cm}^{2} \mathrm{~V}^{-1} \mathrm{~s}^{-1}$ at $300 \mathrm{mK}$. For the microwave measurement the sample temperature was $60 \mathrm{mK}$, measured in the mixing chamber of the dilution refrigerator. We verified that the samples were cooling along with the cryostat down to the reported temperature, by varying the cryostat temperature. d.c. data were taken at $20 \mathrm{mK}$. The data were taken with a growthdirection charge distribution that is approximately symmetric about the well centre with microwave data taken using the set-up in Fig. 1b,c (see Methods). a



b

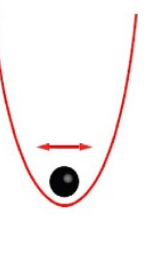



C

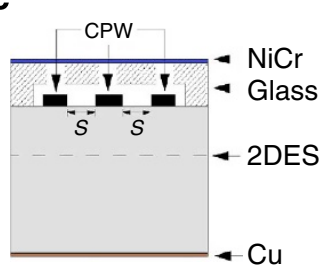

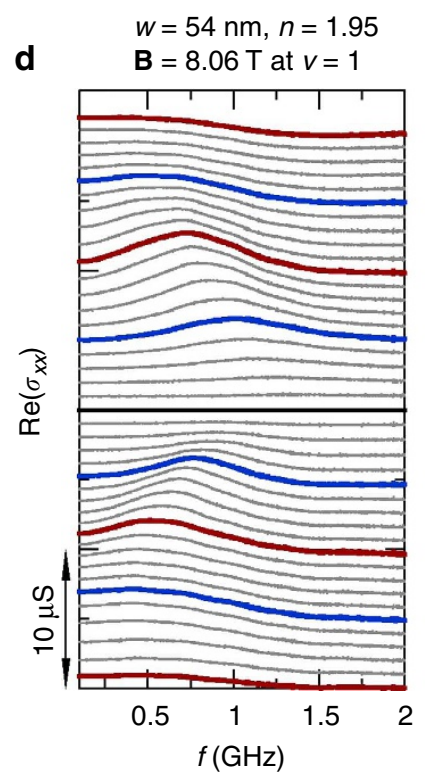


Figure 1 | Microwave spectroscopy of wide quantum wells. (a) Left: sketch of carriers (black) of an electron solid within the residual disorder potential, which is shown by colour (not to scale). Right: a piece (domain) of solid moves within a potential due to the disorder averaged over the domain, giving rise to the pinning mode studied here. (b) Schematic of the microwave set-up. The source and detector are at room temperature. A CPW transmission line is patterned on the top surface of the sample, with metal film of the CPW shown in black. (c) Side view of the sample (not to scale) shows the placement of the front and back gates and the metal film of the CPW. Slots of width s separate the centre conductor and ground planes of the CPW. (d,e) Spectra (diagonal conductivity $\operatorname{Re}\left(\sigma_{x x}\right)$ versus frequency $f$ ) obtained from the $54 \mathrm{~nm}$ well with $n=1.95$ and 2.53 , at several $v^{\star}=v-1$, marked on the right axis of $\mathbf{g}$. Successive spectra are offset upward by $1 \mu \mathrm{S}$ in $\mathbf{d}$ and $2 \mu \mathrm{S}$ in $\mathbf{e}$. (f,g) Spectra for the $65 \mathrm{~nm}$ well at $n=1.39$ and 1.93 offset consecutively by $1 \mu \mathrm{S}$. For each spectrum the magnetic field, $\mathbf{B}$, at $v=1$ is marked. 
$\mathbf{a}$

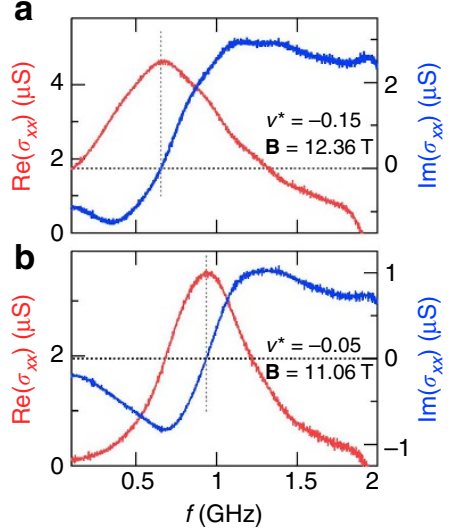

C $\operatorname{Re}\left(\sigma_{x x}\right)(\mu S)$

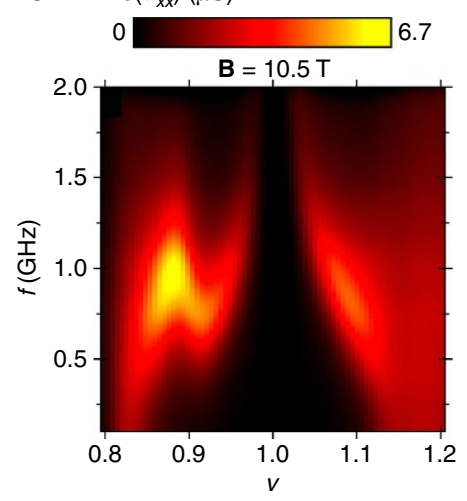

d $\operatorname{Im}\left(\sigma_{x x}\right)(\mu \mathrm{S}) \pm \delta_{\operatorname{lm}}$

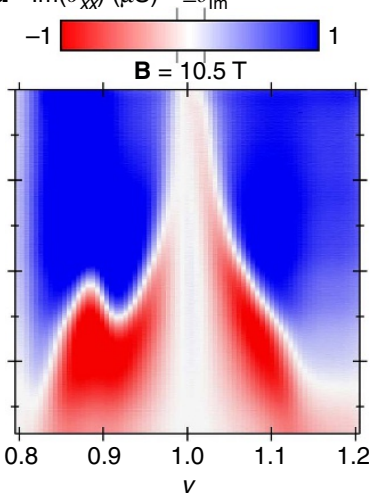

Figure 2 | Resonance peak frequency extraction. (a,b) Real (left axis), and imaginary (right axis), parts of conductivity $\sigma_{x x}$ versus $f$ at two fixed $v^{\star}$ and corresponding $B$ as marked, obtained from the $54 \mathrm{~nm}$ well at $n=2.53$. Dotted vertical lines mark the zero crossing of $\operatorname{Im}\left(\sigma_{x x}\right)$ versus $f$, from which $f_{\mathrm{pk}}$ is determined. The error in $\operatorname{Im}\left(\sigma_{x x}\right), \pm \delta_{\mathrm{Im}}= \pm 0.08 \mu \mathrm{S}$, includes random noise and slower variation in $\operatorname{Im}\left(\sigma_{x x}\right)$ versus $f$ due to residual reflections near the sample mount. (c,d) Colour images in the $(f, v)$ plane for $\operatorname{Re}\left(\sigma_{x x}\right)$ and $\operatorname{Im}\left(\sigma_{x x}\right)$, respectively, the magnetic field value for $v=1$ is marked on the figure. The colour scale in $\mathbf{d}$ is set such that the centre of the white band is 0 , and its extent in $\operatorname{Im}\left(\sigma_{x x}\right)$ is $\pm \delta_{\operatorname{Im}}$. Thus, the white band in the image traces out $f_{\mathrm{pk}}$ versus $v$ and its height is $\pm \delta\left(f_{\mathrm{pk}}\right)$.

a

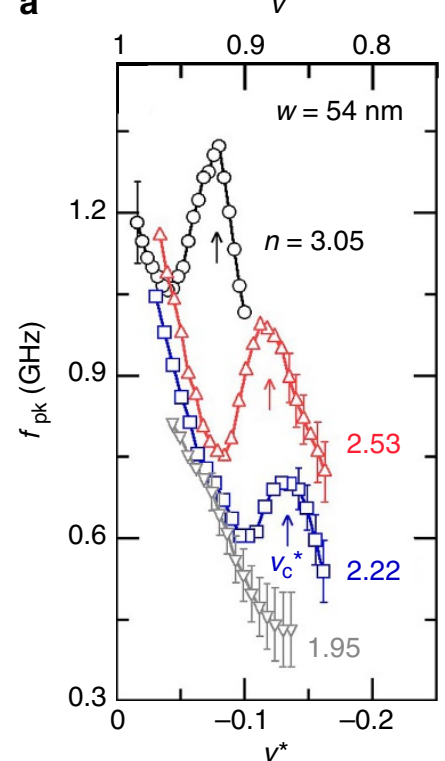

b



Figure 3 | Dependence of resonance peak frequency on filling factor. $f_{\mathrm{pk}}$ versus partial Landau level filling $v^{\star}$ for the $54-\mathrm{nm}(\mathbf{a})$ and $65-\mathrm{nm}$ (b) wells at various carrier densities ( $n$ ) as marked. Arrows mark $v_{c}^{\star}$, the $v^{\star}$ of the local maxima in $f_{\mathrm{pk}}$. Data points without an attached error bar have estimated error, see Fig. 2 for error discussion, in $f_{\mathrm{pk}}$ that is smaller than $\pm 5 \%$.

Microwave spectroscopy. The spectra, diagonal conductivity $\operatorname{Re}\left(\sigma_{x x}\right)$ versus frequency $f$, in Fig. $1 \mathrm{~d}-\mathrm{g}$ give an overview of the evolution of the resonance with Landau filling at different $n$ for the two well widths. We present results in terms of $v^{*}=v-1$, where $\left|v^{*}\right|$ is nearly proportional to the quasiparticle or hole density. In the smaller $n$ states in Fig. 1d,f, and also for the larger $n$ states in Fig. 1e,g with $v^{*}>0$, resonances develop as in ref. 9: as $\left|v^{*}\right|$ increases the resonance forms, develops maximal absorption around $\left|v^{*}\right|=0.08-0.10$ and then fades away, all as $f_{\mathrm{pk}}$ monotonically decreases with $\left|v^{*}\right|$. The resonance development is different for the larger $n$ states with $v^{*}<0$. In Fig. 1e,g $f_{\mathrm{pk}}$ decreases with decreasing $v^{*}$ but near $v^{*} \sim-0.08$ begins to

increase. Further decrease in $v^{\star}$ results in an increase in $f_{\mathrm{pk}}$ until $v^{*} \sim-0.12$, below which $f_{\mathrm{pk}}$ again decreases.

$f_{\mathrm{pk}}$ is extracted from the zero crossing of the imaginary conductivity, $\operatorname{Im}\left(\sigma_{x x}\right)$, versus $f$, as explained in Fig. 2. Figure 3 shows plots of $f_{\mathrm{pk}}$ versus $v^{*}$ at several $n$ for the two WQWs. For the lowest $n$ traces, $f_{\mathrm{pk}}$ versus $\left|v^{*}\right|$ monotonically decreases, but for larger $n$ a local minimum and maximum develop. The minimum and maximum move closer to $v^{\star}=0$ as $n$ increases and are exhibited at lower $n$ for the $65 \mathrm{~nm}$ sample.

We interpret the results in terms of a transition between two distinct solids. Each of the solids exhibits a single pinning mode, and the step in pinning mode frequency, $f_{\mathrm{pk}}$, marks the change from one type of electron solid to the other. At the lowest $n$ for both samples, $f_{\mathrm{pk}}$ monotonically decreases with $\left|v^{*}\right|$. At larger $n$, the $f_{\mathrm{pk}}$ versus $v^{\star}$ curves can be divided into two regions. In the smaller $\left|v^{*}\right|$ regions (closer to $v=1$ ), $f_{\text {pk }}$ versus $v^{*}$ tends towards the lowest $n$ curve, while in the larger $\left|v^{*}\right|$ regions, to the right of the local maximum in Fig. $3, f_{\mathrm{pk}}$ versus $v^{*}$ is enhanced relative to the lowest $n$ curve. We take the enhanced pinning mode frequency $f_{\mathrm{pk}}$ as a characteristic of a solid, which we call S2. It is distinct from S1, which is the only solid seen in the lowest $n$ states of the samples, and which in the larger $n$ states is closer to $v^{*}=0$. With increasing $n$, the transition from S2 to S1 moves closer to $v^{*}=0$, and the transition appears at lower $n$ in the larger $w$ sample. For larger $n$ than those shown in Fig. 3, the step to enhanced $f_{\mathrm{pk}}$ as $\left|v^{*}\right|$ increases can be visible for $v^{*}>0$ also, as is shown in Supplementary Fig. 1. The transition thus appears to occur on both sides of $v=1$; therefore, S2 is favoured by larger $n$, $w$ and $\left|v^{*}\right|$.

Longitudinal transport measurements. We also perform d.c. transport measurements on the same wafers and find that the solid-solid transition picture can underlie features found earlier ${ }^{17}$ in d.c. transport. WQWs were recently found ${ }^{17}$ to exhibit $v$ ranges that are not contiguous with the IQHE minimum centred at $v=1$, but still have Hall resistance quantized at $h / e^{2}$ and vanishing d.c. longitudinal resistance $\left(R_{\mathrm{xx}}\right)$. These regions are called re-entrant integer quantum Hall effects (RIQHEs) ${ }^{21}$ and are due to insulating phases of the partially filled Landau level. At sufficiently high $n$, the RIQHEs appear near $v=4 / 5$ (ref. 17). The RIQHE range extends towards $v=1$ as $n$ is increased by gating, and eventually merges with the main IQHE plateau. The RIQHE 
was ascribed $^{17}$ to a Wigner solid, which can be favoured ${ }^{22}$ over FQHE liquids in WQWs, owing to the softening of the effective electron-electron interaction at short range. The RIQHE sets in for $w / l_{\mathrm{B}} \gtrsim 4$, where $l_{\mathrm{B}}=(h / e B)^{1 / 2}$ is the magnetic length.

Figure 4a,b shows plots of $R_{\mathrm{xx}}$ versus $v^{\star}$ for the 54 and $65 \mathrm{~nm}$ wells at several $n$. Just closer to $v^{\star}=0$ than the minimum due to the RIQHE, the $R_{\mathrm{xx}}$ traces have peaks, both in ref. 17 and in Fig. $4 \mathrm{a}, \mathrm{b}$, which are denoted with arrows. With increasing $n$, the $v^{*}$ of the peak increases as the RIQHE minimum deepens. At the highest $n$, the peak vanishes as the RIQHE minimum merges with the main IQHE minimum. This peak in $R_{\mathrm{xx}}$ could be produced by domain-wall conduction at a phase transition ${ }^{17,23}$.

Microwave spectroscopy and d.c. transport comparison. To directly compare microwave and d.c. measurements, we define
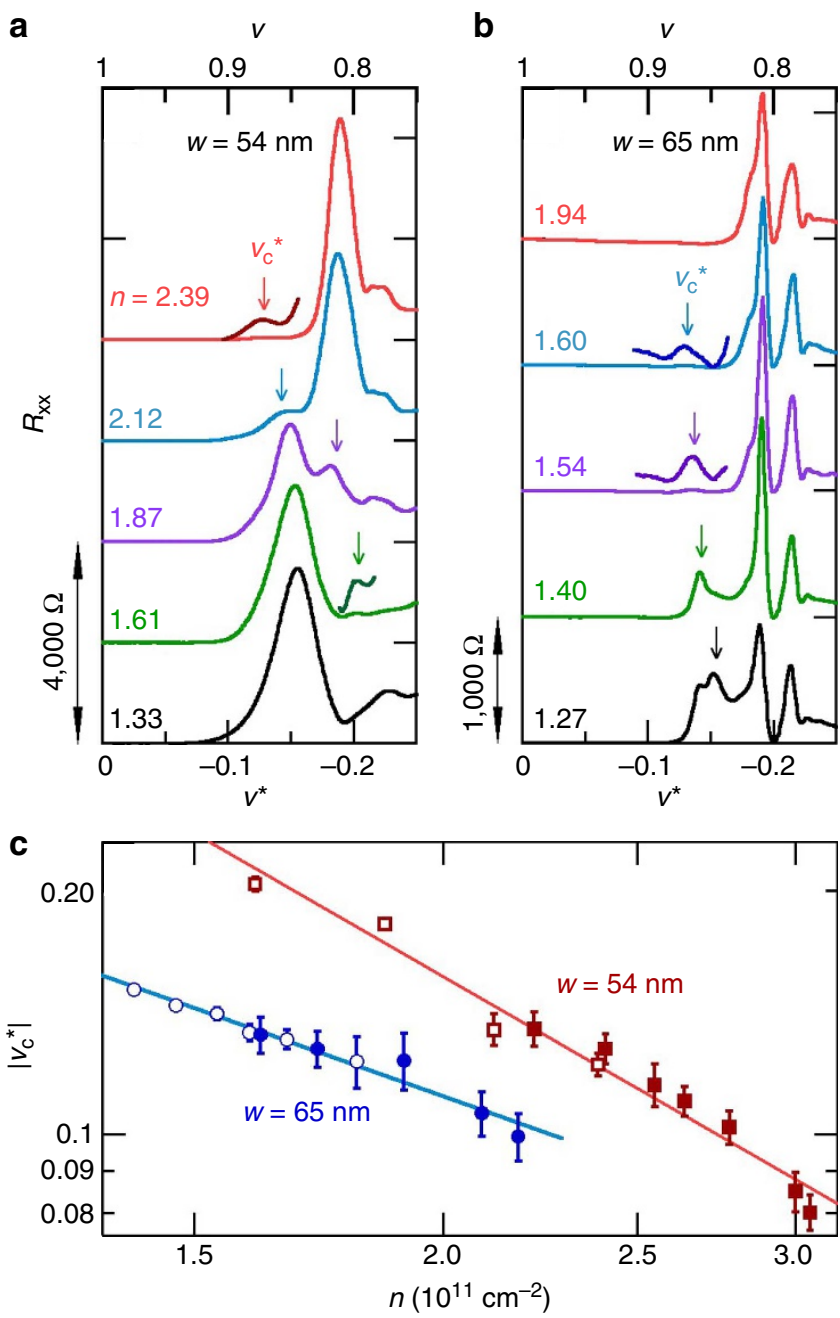

Figure 4 | Longitudinal transport, comparison with microwave resonance spectroscopy. $(\mathbf{a}, \mathbf{b})$ Longitudinal resistance versus partial Landau level filling $v^{\star}$ measured for the $54 \mathrm{~nm}$ (a) and the $65 \mathrm{~nm}$ (b) wells at different densities $n$ as marked, vertically offset for clarity. Arrows mark $v_{c}^{\star}$, the critical partial filling factor. Where $R_{\mathrm{xx}}$ is small, the trace in the region of $v_{\mathrm{c}}^{\star}$ are duplicated and magnified 10 times for clarity. (c) $\left|v_{c}^{*}\right|$ versus $n$ on a loglog scale deduced from d.c. transport measurements (open symbols) and microwave spectra (solid symbols) for the $54 \mathrm{~nm}$ (squares) and $65 \mathrm{~nm}$ (circles). Error bars are based on the width of $v_{c}^{\star}$. Power law fits of the form $v_{c}^{\star} \propto n^{-\alpha}$ are also shown on the plot, where $\alpha=1.35 \pm 0.1$ and $0.87 \pm 0.07$ for the 54 and $65 \mathrm{~nm}$ wells, respectively. the critical filling $v_{\mathrm{c}}^{*}$ as that of the local maximum in the $f_{\mathrm{pk}}$ versus $v^{*}$ measurements and that of the $n$-dependent peak in $R_{\mathrm{xx}}$ versus $v^{*}$, marked by arrows in Figs 3 and 4 . This results in continuous plots of $v_{c}^{*}$ versus $n$, as shown in Fig. $4 \mathrm{c}$, so that S2 can be identified with the d.c. RIQHE. The $v^{*}$ ranges of $v_{c}^{*}$ versus $n$ obtained from microwave and d.c. overlap at intermediate $n$ and are remarkably consistent over the entire $n$ range. Resonances for S2 set in at larger $n$ than required to see the d.c. transport RIQHE, and can be seen at smaller $\left|v^{*}\right|$. When the RIQHE merges with the IQHE the $R_{\mathrm{xx}}$ peak at $v_{\mathrm{c}}^{*}$ is no longer visible, even when the transition is clearly visible in $f_{\mathrm{pk}}$. Pinning modes are not visible in the RIQHE ranges at larger $\left|v^{*}\right|$, possibly because uncondensed carriers are present, which dampen the resonance.

\section{Discussion}

Chen et al. ${ }^{10}$ reported evolution of the pinning mode with $v$ below the termination of the FQHE series, in quantum wells with $w=50$ and $65 \mathrm{~nm}$, but with much lower $n(\simeq 1$ and 0.5 , respectively) than those studied here. For $0.12<v<0.18$, the spectra appeared irregular with multiple peaks, although welldefined peaks appeared above and below that range. In ref. 10 that $v$ range was the same in each sample, and for $v \gtrsim 0.18$, the resonance was wave vector $(q)$-dependent. The $q$ dependence was found by comparing transmission lines of different slot width, $s$, $q \sim \pi / s$ (see Methods). Checking for $q$ dependence in the present $54 \mathrm{~nm}$ WQW sample using $s=80 \mu \mathrm{m}$ we found none, with excellent agreement between the $s=80 \mu \mathrm{m} f_{\mathrm{pk}}$ versus $v^{*}$ and the $s=30 \mu \mathrm{m}$ data shown in Fig. 3a.

Since a pinning mode is identified with electron solids, an enhanced $f_{\mathrm{pk}}$ similar to that of $\mathrm{S} 2$ relative to $\mathrm{S} 1$ is because of better 'contact' between the carriers of the solid and the disorder, increasing the effective pinning. This can be because of a smaller $C_{\mathrm{t}}$ in $\mathrm{S} 2$ than in $\mathrm{S} 1$, as mentioned above, but the disorder contact can also be increased by a change in the charge distribution of each carrier in the lattice or in the lattice type (for example, from one-component to two-component).

Theories, 24-26 have considered crystals of composite fermions ${ }^{27}$, which are categorized by the number, $2 \mathrm{p}$, of vortices bound to a carrier. Theory predicts a series of distinct composite fermion Wigner solid ( ${ }^{2} \mathrm{P}$ CFWS) phases with $2 \mathrm{p}$ increasing as $v$ decreases ${ }^{9,24}$. The transition from $2 \mathrm{p}=2-4$ occurs as $v$ goes from $1 / 5$ to $1 / 6$. Although ${ }^{4} C_{\mathrm{t}}$ is predicted to soften near the transition, well within the ${ }^{4}$ CFWS it is calculated to be a factor of $\sim 2$ larger than ${ }^{2} C_{\mathrm{t}}$ and would result in a lower $f_{\mathrm{pk}}$ in the ${ }^{4}$ CFWS. Identifying S2 as a ${ }^{2}$ CFWS and S1 as a ${ }^{4}$ CFWS would be consistent with the shear moduli predicted by ref. 7 . However, ref. 7 predicts little sensitivity of the phase diagram to $w$, but we do observe dependence of $v_{c}^{*}$ on $w$ and $n$.

Are there other possible interpretations for the two solids? A two-component bilayer Wigner solid can exist under certain conditions in a WQW ${ }^{18,19}$, particularly if the sub-band gap, $\Delta$, is small enough relative to the Coulomb energy, $E_{\mathrm{c}}$. Such a twocomponent Wigner solid could have increased disorder and $f_{\mathrm{pk}}$ because of charge being pushed closer to the interfaces ${ }^{12}$ and would be favoured by larger $\left|v^{*}\right|$, giving smaller carrier spacing, and by larger $w$. For the $54 \mathrm{~nm}$ well with typical $v_{\mathrm{c}}=-0.1$, $n=2.8$, we find from simulations ${ }^{16,18}$ that $\Delta \sim 16 \mathrm{~K}$, which according to ref. 28 is about three times too large for a twocomponent lattice to form.

A composite fermion ground-state spin transition ${ }^{29,30}$ is also unlikely to explain the observed phenomena. The composite fermion spin-Landau levels only cross above the Fermi level ${ }^{30}$. Although skyrmion solid formation has been reported ${ }^{31}$ from pinning modes near $v=1$, at the larger $v_{\mathrm{c}}^{*}$ studied here large quasihole density would suppress skyrmion effects ${ }^{32}$. 
In summary, near $v=1$ in WQWs, we have found a change in $f_{\mathrm{pk}}$ that is naturally interpreted as signature of a transition between two different solids. S2, which exists at larger $\left|v^{\star}\right|$, larger $n$ and larger $w$, has enhanced $f_{\mathrm{pk}}$ relative to the other phase. While the origin of the transition remains unclear, the possibilities, particularly of a transition in CFWS vortex number, are of fundamental importance.

\section{Methods}

Charge distribution. For d.c. measurements, the symmetry of the charge distribution about the well centre was maintained using the Fourier transform of $R_{\mathrm{xx}}$ versus $\mathbf{B}$ in the Shubnikov-de Haas regime to minimize the gap, $\Delta$, between the lowest and first excited sub-bands ${ }^{16,20}$. For the microwave measurements, balance of the change between front and back halves of the well was maintained by biasing front and back gates such that individually each would change the carrier density by equal amounts.

Microwave spectroscopy technique. Our microwave spectroscopy ${ }^{2}$ technique ${ }^{9,10,31}$ uses a coplanar waveguide (CPW) on the surface of a sample. A $\mathrm{NiCr}$ front gate was deposited on glass that was etched to space it from the CPW by $\sim 10 \mu \mathrm{m}$. A schematic diagram of the microwave measurement technique is shown in the left panel of Fig. 1b and side view of the sample is shown in Fig. 1c. The microwave electronics, consisting of a pre-amplifier, network analyser (Agilent E5071 C) and attenuators, was entirely located at room temperature. Semi-rigid coaxial cables, including both copper and superconducting types, were used within the cryostat to connect the sample to room-temperature ports at the cryostat top. The connections are carefully optimized to minimize reflections, and the incident power on the sample fluctuates only $\sim 10 \%$ because of standing waves on the coaxial cables. The effects of these standing waves, and of the frequency-dependent loss of the cables, were largely removed by taking the ratio $t / t_{0}$, where $t$ was the amplitude at the receiver and $t_{0}$ is the amplitude for $v=1$. We calculate the diagonal conductivity as $\sigma_{\mathrm{xx}}(f)=\left(s / l Z_{0}\right) \ln \left(t / t_{0}\right)$, where $s=30 \mu \mathrm{m}$ is the distance between the centre conductor and ground plane, $l=28 \mathrm{~mm}$ is the length of the $\mathrm{CPW}$ and $Z_{0}=50 \Omega$ is the characteristic impedance without the 2DES. Hence, $\sigma_{x x}(f)$ is the difference between the conductivity and that for $v=1$; just at $v=1$ the conductivity is vanishing at low temperature. The microwave measurements were carried out in the low-power limit, where the measurement is not sensitive to the excitation power.

Longitudinal transport measurements. For d.c. transport measurements, no transmission line was used; measurements were performed in a van der Pauw geometry using standard $(\simeq 30 \mathrm{~Hz})$ lock-in technique, with front and back gates both deposited directly on the sample surfaces.

\section{References}

1. Lozovik, Y. E. \& Yudson, V. Crystallisation of a two dimensional electron gas in magnetic field. JETP Lett. 22, 11-12 (1975).

2. Andrei, E. Y. et al. Observation of a magnetically induced Wigner solid. Phys. Rev. Lett. 60, 2765-2768 (1988).

3. Goldman, V. J., Santos, M., Shayegan, M. \& Cunningham, J. E. Evidence for two-dimensional quantum Wigner crystal. Phys. Rev. Lett. 65, 2189-2192 (1990).

4. Jiang, H.-W. et al. Quantum liquid versus electron solid around $\mathrm{v}=1 / 5$ Landau-level filling. Phys. Rev. Lett. 65, 633-636 (1990).

5. Williams, F. I. B. et al. Conduction threshold and pinning frequency of magnetically induced Wigner solid. Phys. Rev. Lett. 66, 3285-3288 (1991).

6. Yang, K., Haldane, F. D. M. \& Rezayi, E. H. Wigner crystals in the lowest Landau level at low-filling factors. Phys. Rev. B 64, 081301 (2001).

7. Archer, A. C., Park, K. \& Jain, J. K. Nature of the crystal phase between $1 / 5$ and 2/9 fractional Hall liquids. Phys. Rev. Lett. 111, 146804 (2013).

8. Shayegan, M. in Perspectives in Quantum Hall Effects. (eds Das Sarma, S. \& Pinczuk, A.) 343 (Wiley-Interscience, 1997).

9. Chen, Y. et al. Microwave resonance of the 2D Wigner crystal around integer Landau fillings. Phys. Rev. Lett. 91, 016801 (2003).

10. Chen, Y. P. et al. Evidence for two different solid phases of two-dimensional electrons in high magnetic fields. Phys. Rev. Lett. 93, 206805 (2004).

11. Fukuyama, H. \& Lee, P. A. Pinning and conductivity of two-dimensional charge-density waves in magnetic fields. Phys. Rev. B 18, 6245-6252 (1978).

12. Fertig, H. A. Electromagnetic response of a pinned Wigner crystal. Phys. Rev. B 59, 2120-2141 (1999).

13. Chitra, R., Giamarchi, T. \& Le Doussal, P. Pinned Wigner crystals. Phys. Rev. B 65, $035312(2001)$

14. Fogler, M. M. \& Huse, D. A. Dynamical response of a pinned two-dimensional Wigner crystal. Phys. Rev. B 62, 7553-7570 (2000).
15. Li, C.-C. et al. Microwave resonance and weak pinning in two-dimensional hole systems at high magnetic fields. Phys. Rev. B 61, 10905-10909 (2000).

16. Liu, Y., Shabani, J. \& Shayegan, M. Stability of the $q / 3$ fractional quantum Hall states. Phys. Rev. B 84, 195303 (2011).

17. Liu, Y. et al. Observation of reentrant integer quantum Hall states in the lowest Landau level. Phys. Rev. Lett. 109, 036801 (2012).

18. Shayegan, M., Manoharan, H. C., Suen, Y. W., Lay, T. S. \& Santos, M. B. Correlated bilayer electron states. Semicond. Sci. Technol. 11, 1539-1545 (1996).

19. Manoharan, H. C., Suen, Y. W., Santos, M. B. \& Shayegan, M. Evi dence for a bilayer quantum Wigner solid. Phys. Rev. Lett. 77, 1813-1816 (1996).

20. Suen, Y. W., Manoharan, H. C., Ying, X., Santos, M. B. \& Shayegan, M. Origin of the $\mathrm{v}=1 / 2$ fractional quantum Hall state in wide single quantum wells. Phys. Rev. Lett. 72, 3405-3408 (1994).

21. Lilly, M. P. et al. Evidence for an anisotropic state of two-dimensional electrons in high Landau levels. Phys. Rev. Lett. 82, 394-397 (1999).

22. Price, R., Zhu, X., Das Sarma, S. \& Platzman, P. M. Laughlin-liquid Wigner-solid transition at high density in wide quantum wells. Phys. Rev. B 51, 2017-2020 (1995).

23. Gervais, G. et al. Competition between a fractional quantum Hall liquid and bubble and Wigner crystal phases in the third Landau level. Phys. Rev. Lett. 93, 266804 (2004).

24. Chang, C.-C., Jeon, G. S. \& Jain, J. K. Microscopic verification of topological electron-vortex binding in the lowest Landau-level crystal state. Phys. Rev. Lett. 94, 016809 (2005).

25. Narevich, F., Murthy, G. \& Fertig, H. A. Hamiltonian theory of the composite-fermion Wigner crystal. Phys. Rev. B 64, 245326 (2001).

26. Yi, H. \& Fertig, H. A. Laughlin-Jastrow-correlated Wigner crystal in a strong magnetic field. Phys. Rev. B 58, 4019-4027 (1998).

27. Jain, J. K. Composite Fermions (Cambridge University Press, 1999).

28. Narasimhan, S. \& Ho, T.-L. Wigner-crystal phases in bilayer quantum Hall systems. Phys. Rev. B 52, 12291-12306 (1995).

29. Du, R. R. et al. Fractional quantum Hall effect around $v=3 / 2$ : Composite fermions with a spin. Phys. Rev. Lett. 75, 3926-3929 (1995).

30. Yeh, A. S. et al. Effective mass and g factor of four-flux-quanta composite fermions. Phys. Rev. Lett. 82, 592-595 (1999).

31. Zhu, H. et al. Pinning-mode resonance of a skyrme crystal near Landau-Level filling factor $\mathrm{v}=1$. Phys. Rev. Lett. 104, 226801 (2010).

32. Cote, R. et al. Collective excitations, NMR, and phase transitions in Skyrme crystals. Phys. Rev. Lett. 78, 4825-4828 (1997).

\section{Acknowledgements}

We thank Jainendra Jain for illuminating discussions. The microwave spectroscopy work at NHMFL was supported through DOE grant DE-FG02-05-ER46212 at NHMFL/FSU. The National High Magnetic Field Laboratory (NHMFL) is supported by NSF Cooperative Agreement No. DMR-0654118 by the State of Florida and by the DOE. The work at Princeton was funded through the NSF (grants DMR-1305691 and MRSEC DMR-0819860), the Keck Foundation and the Gordon and Betty Moore Foundation (grant GBMF2719).

\section{Author contributions}

A.T.H. performed the microwave measurements, analysed the data and co-wrote the manuscript. Y.L. designed, performed and analysed the d.c. experiments and did numerical simulations. B.A.M. and B.H.M. performed initial microwave measurements L.W.E. and M.S. conceived and designed the experiment, discussed data analysis and cowrote the manuscript. L.N.P., K.W.W. and K.W.B. were responsible for the growth of the samples.

\section{Additional information}

Supplementary Information accompanies this paper at http://www.nature.com/ naturecommunications

Competing financial interests: The authors declare no competing financial interests.

Reprints and permission information is available online at http://npg.nature.com/ reprintsandpermissions/

How to cite this article: Hatke, A. T. et al. Microwave spectroscopic observation of distinct electron solid phases in wide quantum wells. Nat. Commun. 5:4154 doi: $10.1038 /$ ncomms5154 (2014).

This work is licensed under a Creative Commons AttributionNonCommercial-NoDerivs 4.0 International License. The images or other third party material in this article are included in the article's Creative Commons license, unless indicated otherwise in the credit line; if the material is not included under the Creative Commons license, users will need to obtain permission from the license holder to reproduce the material. To view a copy of this license, visit http:// creativecommons.org/licenses/by-nc-nd/4.0/ 